\title{
Impact of Wind Power Variability on Sub-transmission Networks
}

\author{
Sina Sadeghi Baghsorkhi, Student Member, IEEE
}

\author{
Ian A. Hiskens, Fellow, IEEE
}

\begin{abstract}
The inherent variability of wind power injections becomes particularly important for wind farms connected to weaker networks, as voltage deviations become more significant and voltage regulation more challenging. Through sensitivity analysis and continuation methods, this paper examines the impact of wind injection on transformer tap operation in subtransmission networks. The results of a tap operation simulation show that voltage regulation at wind injection nodes increases tap change operations. The trade-off between local voltage regulation and tap change frequency is fundamentally important in optimizing the size of reactive compensation used for voltage regulation at wind injection nodes.
\end{abstract}

Index Terms-Wind power; voltage regulation; transformer tap changing; continuation methods; sensitivity analysis.

\section{INTRODUCTION}

$\mathbf{I}$ $\mathrm{T}$ is generally more convenient and economical to connect distributed generation, in particular wind farms, at subtransmission levels $(40-120 \mathrm{kV})$. In fact, in many cases it is unavoidable as only a lower voltage network is available. However, relative weakness of the sub-transmission network, characterized by low short-circuit ratios and high impedances [1], poses serious challenges for the large-scale integration of variable generation. In the case of wind power, its inherent variability can lead to unusual reactive power absorption and injection patterns. Since reactive power is closely coupled with voltage magnitudes and voltage regulating assets, wind power variations at multiple nodes may affect bus voltages and transformer tap positions in complicated and unexpected ways.

Many utilities require wind-farm operators to regulate the voltage at the point of interconnection (POI) to a setpoint value determined by the system operator. Nevertheless, varying power flow can cause voltage fluctuation at unregulated load buses that are sometimes located far from the POI. As voltage fluctuations can be problematic for consumers, utilities are required to install additional voltage regulating equipment. Varying power flow can also result in a higher frequency of tap-changing operation of transformers that connect weak sub-transmission networks to the transmission system. This increase is unacceptable for asset owners as it may significantly shorten the lifetime of these expensive devices.

Therefore it is important to examine interactions between wind-farm voltage regulation and other, pre-existing voltage regulating equipment, such as tap-changing transformers, and coordinate their goals to achieve optimal operation of the grid. Previous studies have shown for radial distribution feeders that

Research supported by the Department of Energy through grant DEEE0001382.

Sina Sadeghi Baghsorkhi (sinasb@umich.edu) and Ian A. Hiskens (hiskens@umich.edu) are with the Department of Electrical Engineering and Computer Science, University of Michigan, Ann Arbor, Michigan. voltage regulation at the POI tends to increase tap-changing operations [2]. However, many sub-transmission and distribution networks have a mesh structure. The presence of multiple wind injection nodes in a mesh network further complicates the impact of wind on voltages and taps. Existing techniques for analyzing power system response provide limited insights into the complex interactions between regulating devices and variable generation.

This paper examines the impact of wind generation on subtransmission networks, focusing particularly on the effect of power flow variability on the voltage profile and transformer tap-changing. Studies are based on a mesh network that serves the south-eastern region of Michigan. It is anticipated that major wind developments will occur in this region, and that the wind farms will be connected to the $40 \mathrm{kV}$ sub-transmission network. We have used sensitivity analysis, based on the power flow Jacobian, to identify transformers that are most sensitive to wind-power variability at two sub-transmission wind generation nodes. Power flow continuation methods are also used to produce contour diagrams that allow more extensive investigations of the quantities that are most sensitive to wind injections at these two nodes.

The use of the power flow Jacobian for sensitivity analysis is not new, with applications dating back to the late 1960s [3]. Since then, sensitivity analysis has been applied in a wide range of power system studies, from voltage stability [4], [6], [5] to assessing the impact of distributed generation on line losses [7]. Continuation methods have been in existence for many years [8], and first found application in power system studies in the early 1980s, see for example [9]. Subsequent power system applications of continuation methods include voltage stability studies [10] and solution space investigations [11]. We will use these methods to examine the impact of active power injection on tap positions and voltage magnitudes in the context of wind power.

The paper is structured as follows. Section II describes the analysis tools used to investigate the impact of wind injection. Section III applies these tools to the network of interest, explains various observed patterns, and establishes general trends in voltage regulation and tap-changer operation in weak sub-transmission systems. The temporal characteristics of tapchanger operation are discussed in Section IV, and conclusions are provided in Section V.

\section{Power System AnAlysis Tools}

\section{A. Sensitivity Analysis}

The power flow equations can be expressed in the form

$$
\begin{aligned}
& P(\theta, V)=0 \\
& Q(\theta, V)=0
\end{aligned}
$$


where (1) describes the active power balance at PV and PQ buses, (2) describes the reactive power balance at PQ buses, $\theta$ is the vector of voltage angles (relative to the slack bus) at all PV and PQ buses, and $V$ is the vector of voltage magnitudes at PQ buses [12]. It follows that $P$ and $\theta$ have equal dimensions, and likewise $Q$ and $V$ have the same dimension.

Transformer taps can be incorporated into the power flow equations by assuming tap positions $a_{i}$ are continuous variables, and noting that each transformer regulates a particular bus voltage. That bus voltage magnitude $V_{i}$ takes on a known fixed value, and so it can be replaced in (1)-(2) by the new variable $a_{i}$. The power flow equations can be generalized accordingly by replacing the voltage vector $V$ with $\mathcal{V}=\left[\begin{array}{l}\tilde{V} \\ a\end{array}\right]$ where $\tilde{V}$ is the vector of voltage magnitudes at non-regulated buses, and $a$ is the vector of tap positions associated with the transformers that are regulating bus voltages. Note that $Q$ and $\mathcal{V}$ still have equal dimensions.

Taking partial derivatives of $P$ and $Q$ with respect to $\theta$ and $\mathcal{V}$ gives the linearized relationship,

$$
\left[\begin{array}{c}
\Delta P \\
\Delta Q
\end{array}\right]=\left[\begin{array}{ll}
P_{\theta} & P_{\mathcal{V}} \\
Q_{\theta} & Q_{\mathcal{V}}
\end{array}\right]\left[\begin{array}{c}
\Delta \theta \\
\Delta \mathcal{V}
\end{array}\right]
$$

where $P_{\theta} \equiv \frac{\partial P}{\partial \theta}$, and likewise for the other sub-matrices. We are interested in how variations $\Delta P$ in the injected active power at wind-farm locations affect voltage magnitudes and tap positions, which are given by $\Delta \mathcal{V}=\left[\begin{array}{c}\Delta \tilde{V} \\ \Delta a\end{array}\right]$. For transmission systems, where resistance is negligible, the offdiagonal blocks in (3) are almost zero, and so are normally neglected. This decouples $\Delta P$ from $\Delta \mathcal{V}$. However in subtransmission and distribution networks, where resistance is non-negligible, the off-diagonal blocks become important. To understand how $\Delta \mathcal{V}$ varies with $\Delta P$, we can use the Matrix Inversion Lemma [13] to give,

$$
\begin{aligned}
\Delta \mathcal{V}= & -\left[Q_{\mathcal{V}}-Q_{\theta} P_{\theta}^{-1} P_{\mathcal{V}}\right]^{-1} Q_{\theta} P_{\theta}^{-1} \Delta P \\
& +\left[Q_{\mathcal{V}}-Q_{\theta} P_{\theta}^{-1} P_{\mathcal{V}}\right]^{-1} \Delta Q .
\end{aligned}
$$

In our analysis, we assume that reactive power remains unchanged at PQ buses, so $\Delta Q=0$. It follows that the desired sensitivities are given by,

$$
\begin{aligned}
\Delta \mathcal{V} & =-\left[Q_{\mathcal{V}}-Q_{\theta} P_{\theta}^{-1} P_{\mathcal{V}}\right]^{-1} Q_{\theta} P_{\theta}^{-1} \Delta P \\
& =S \Delta P .
\end{aligned}
$$

where $S$ has the structure $S=\left[\begin{array}{c}S_{\tilde{V}} \\ S_{a}\end{array}\right]$, and

$\Delta \tilde{V}=S_{\tilde{V}} \Delta P$ for buses where voltages are not regulated, $\Delta a=S_{a} \Delta P$ for transformer taps.

\section{B. Power Flow Continuation}

Sensitivity values only provide local information around a single operating point. This can be helpful in identifying bus voltages and transformer taps that are highly sensitive to wind injection. However this should be verified by a more complete view of the behavior of these variables for larger changes.

The power flow equations (1)-(2) can be written in generalized form as $f(x)=0$, where $f$ and $x$ have the same dimension. This problem is fully determined, so solutions will be points. If a single parameter is allowed to vary, for example the active power at a PV or PQ bus, or the voltage setpoint at a PV bus, the problem takes the form,

$$
f(x, \lambda)=0,
$$

where $\lambda$ is the single free parameter. Now the problem has one more variable than constraint, so is under-determined. In this case, the solution is no longer a single point, but rather defines a curve. Freeing a second parameter results in a surface which can be shown as a collection of curves (i.e. contour diagram), similar to a topographic map with contours of elevation. This concept underlies the continuation power flow.

Continuation methods for solving problems of the form (6) are well documented [8], [14]. Predictor-corrector algorithms, such as the Euler homotopy method, provide a robust process for obtaining a sequence of points along the desired curve. Application of this particular method to power system problems is discussed in [9], [11].

\section{GRID ANALYSIS}

Figure 1 shows a section of the power grid that is being studied for wind development. Active power is delivered from central power plants to the $40 \mathrm{kV}$ sub-transmission system through a $120 \mathrm{kV}$ transmission loop that is highlighted in red. At the interconnection points, the lower voltage $(40 \mathrm{kV}$ side) is regulated by five tap changing transformers highlighted by red ellipses. We have modeled two wind injection nodes, highlighted in blue, each with a capacity 50 MW. Typically distribution companies require wind farm operators to regulate the voltage at the point of interconnection to its setpoint value, and this makes reactive power compensation ${ }^{1}$ at the wind injection nodes indispensable.

To better understand the impact of this local reactive compensation on transformer tap operation and voltage magnitudes of adjacent nodes, throughout this section and the following we have contrasted the following two cases:

1) No compensation at wind injection nodes.

2) Compensation of \pm 15 MVAr at each wind injection node.

The case of no compensation is often not permitted by the system operator as voltage fluctuations caused by wind variability place enormous stress on voltage regulating equipment in distribution substations. Figure 2(a) shows the voltage contour diagram of load bus $L_{1}$ in the vicinity of the wind injection nodes (highlighted in green in Figure 1). The region of interest, corresponding to the feasible range of wind injection at each node, is highlighted in the red box, and an enlarged version is shown in Figure 2(b). As wind injection at the nodes simultaneously varies from 0 to $50 \mathrm{MW}$, voltage increases from 0.99 p.u. to 1.10 p.u. This voltage rise phenomenon, caused by wind-power injection on weak networks [15], has lead to stricter grid standards requiring large distributed-generation installations to operate in voltage control mode. Figure 3 shows voltage contours of the same bus for the case of \pm 15 MVAr compensation and, as shown in Figure 3(b), the voltage fluctuation range is now reduced to 0.99-1.05 p.u. However our study illustrates later that even

\footnotetext{
${ }^{1}$ Statcoms are often used to provide dynamic reactive support.
} 


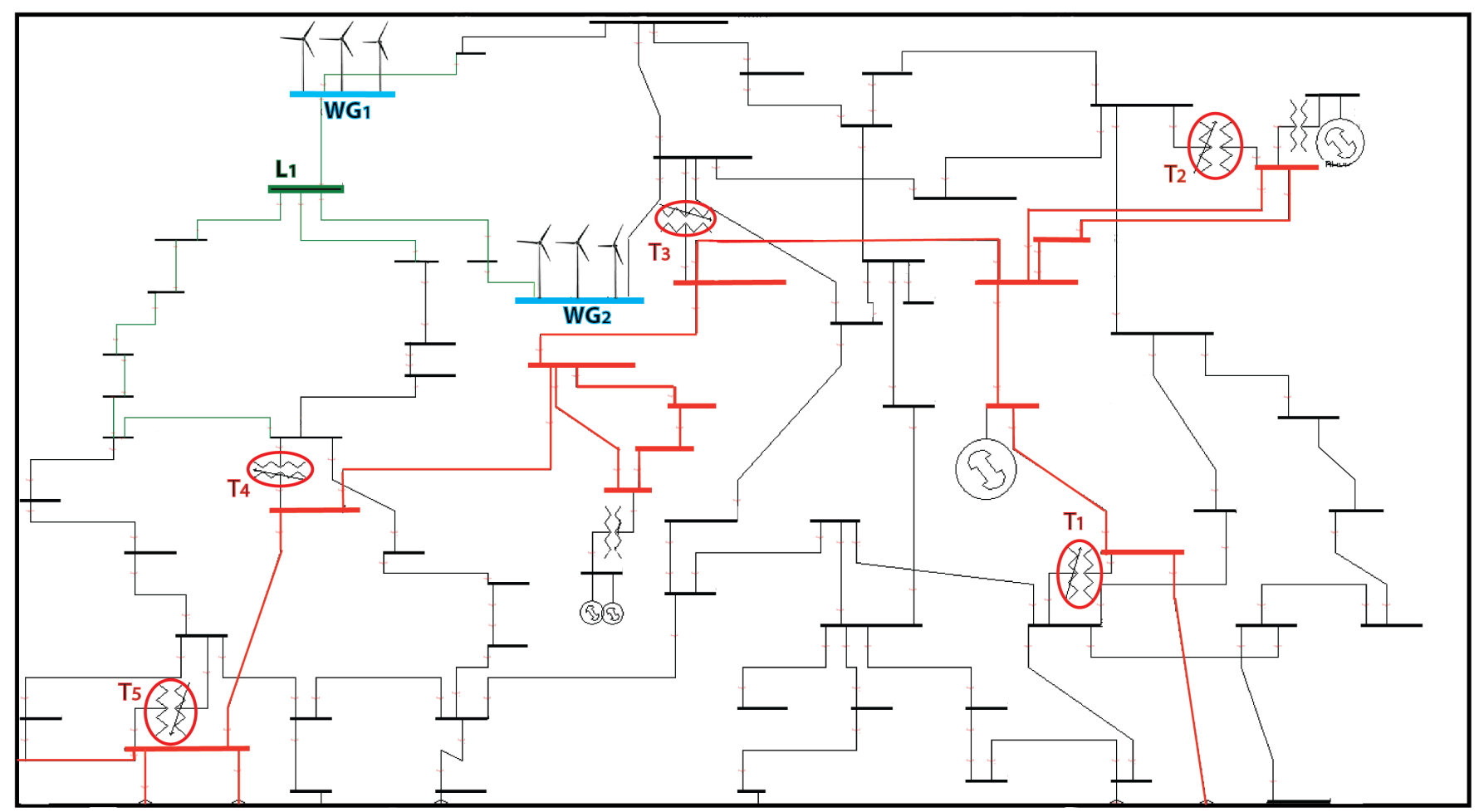

Fig. 1. Wind development network: wind injection nodes $W G_{1}$ and $W G_{2}$ are highlighted in blue, load bus $L_{1}$ in green, and tap changing transformers in red.

though the no-compensation case may appear extreme and unpractical, it is useful in the way it highlights the need for coordination between reactive compensation at wind injection nodes and transformer tap operation.

The transformer tap sensitivity values for the two cases mentioned above are presented in Tables I and II respectively. We have considered the three operating points of no wind, $40 \%$ and $80 \%$ wind output, and assumed $100 \%$ correlation between wind injections. The latter two operating points correspond to medium and high wind generation respectively. It can be noticed that tap positions are in general more sensitive to wind injection in the case of \pm 15 MVAr reactive compensation and that $T_{3}$ has the highest sensitivity values followed by $T_{4}$ and $T_{5}$. Furthermore, in the case of \pm 15 MVAr reactive compensation, tap sensitivity is high at no wind, peaks at medium wind and drops to its lowest at high wind. This pattern is most visible for $T_{3}$ and $T_{4}$. In contrast, the no-compensation sensitivity values are highest at no wind and are almost zero at medium and high wind.

These sensitivity values provide us with an indication as to which transformers and buses are most sensitive and at what range of wind injection. With that knowledge we can generate the contour diagrams of relevant variables (tap positions or voltage magnitudes). Figure 4(a) shows the tap position contour diagram of $T_{3}$ which overall had the highest sensitivity values. By looking at the region of interest shown in Figure 4(b), we see that at low to medium wind injection, tap contour lines (each corresponding to a tap step) are closely spaced whereas at higher wind the tap position does not change at all. Contrasting Figures 3(b) and 4(b) reveals an interesting pattern. Regions of high tap and low voltage sensitivity almost entirely overlap.

Moreover there are discontinuities in the contour lines when injection is high at one node and low at the other. These somewhat symmetrical discontinuity points correspond to limits of reactive compensation at each injection node. As wind injection increases, reactive compensation absorbs more reactive power until its inductive limit is encountered. As wind injection continues to increase, the voltage magnitude of the injection node and adjacent nodes starts to rise. Figures 5 and 6 show the contour lines corresponding to inductive limits of $W G_{1}$ and $W G_{2}$ superimposed on voltage magnitude and tap position diagrams respectively. The solution space is divided into four regions labelled $\mathrm{A}, \mathrm{B}, \mathrm{C}$ and $\mathrm{D}$. In region $\mathrm{A}$, both $W G_{1}$ and $W G_{2}$ are regulating. In region $\mathrm{C}, W G_{2}$ has reached its inductive limit but $W G_{1}$ is still regulating, whereas in region $\mathrm{D}$ the situation is the reverse. In region $\mathrm{B}$ both $W G_{1}$ and $W G_{2}$ have reached their inductive limits. This latter region corresponds with where $T_{3}$ tap position stabilizes. According to the sensitivity values, this is also the case for all other transformers. The tap position contour diagram of $T_{3}$ for the case with no compensation, shown in Figure 7, also confirms, albeit in a negative way, the observation that reactive compensation results in higher tap sensitivity. Therefore, in the absence of any voltage regulation at the injection nodes, tap position sensitivity is reduced.

However, before concluding that there exists a trade-off between voltage regulation at the injection nodes and tap change operation, we need to investigate the actual tapchanging operation of transformers under normal operating conditions, prior to wind. This base case, where transformer taps are adjusted only in response to changes in the system 


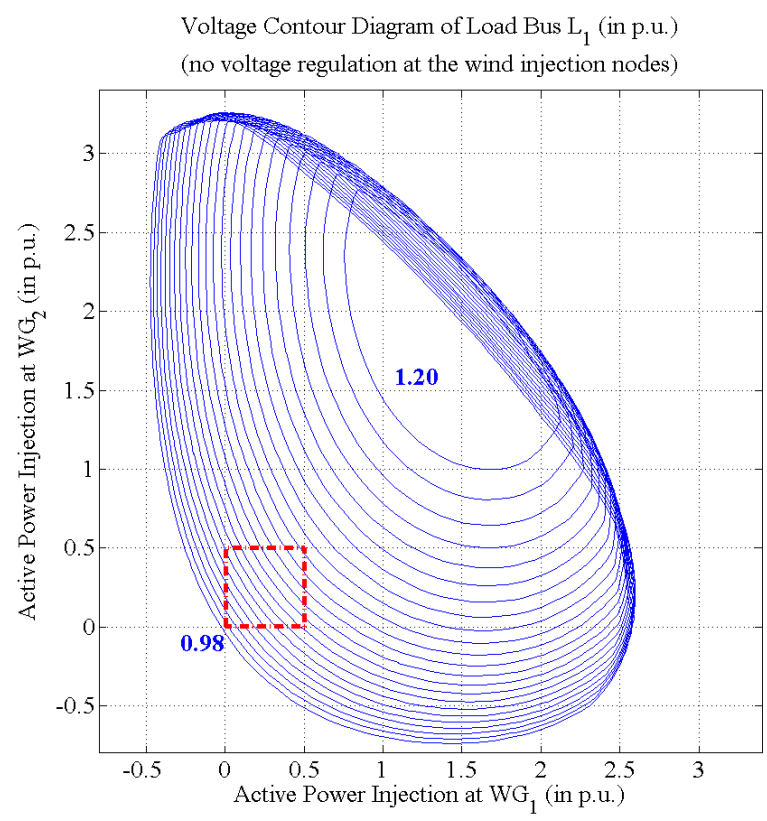

(a) Global

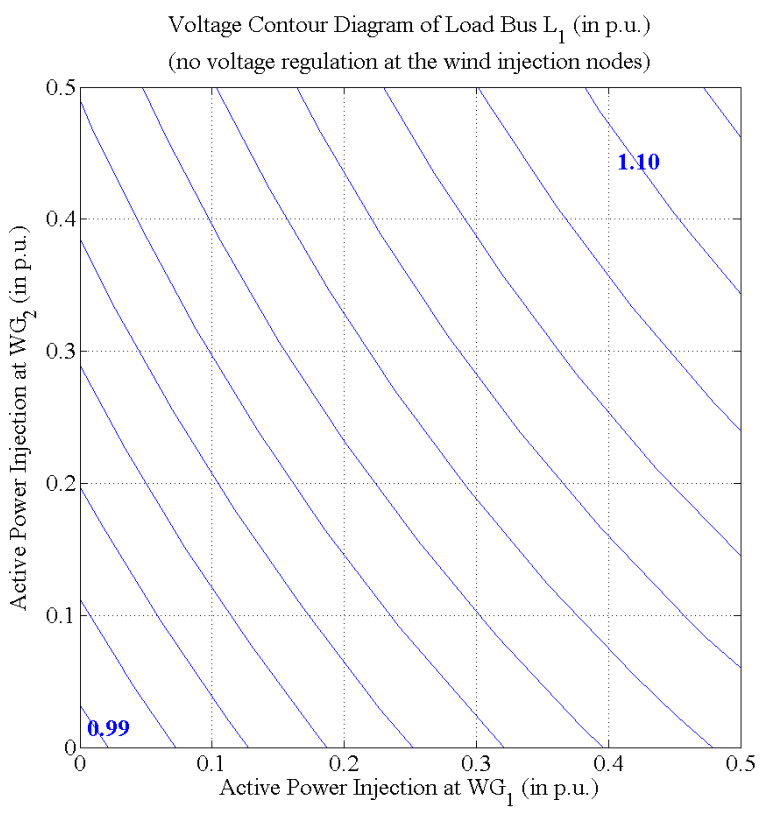

(b) Region of Interest

Fig. 2. Contour diagrams of voltage magnitude at load bus $L_{1}$ for varying active power injections at wind nodes (no voltage regulation).

load, must be compared with cases where variable wind is added, with and without local reactive compensation.

\section{Wind And TAP Change OPERATion Simulation}

The previous section established a relationship between voltage regulation of wind injection nodes and sensitivity of the tap positions of the transformers that connect the subtransmission and transmission systems. This will be explored further via simulation of the tap change operation of the five transformers in the system of interest.

The simulation is based on power flow code developed in Matlab and verified with commercial power flow programs.

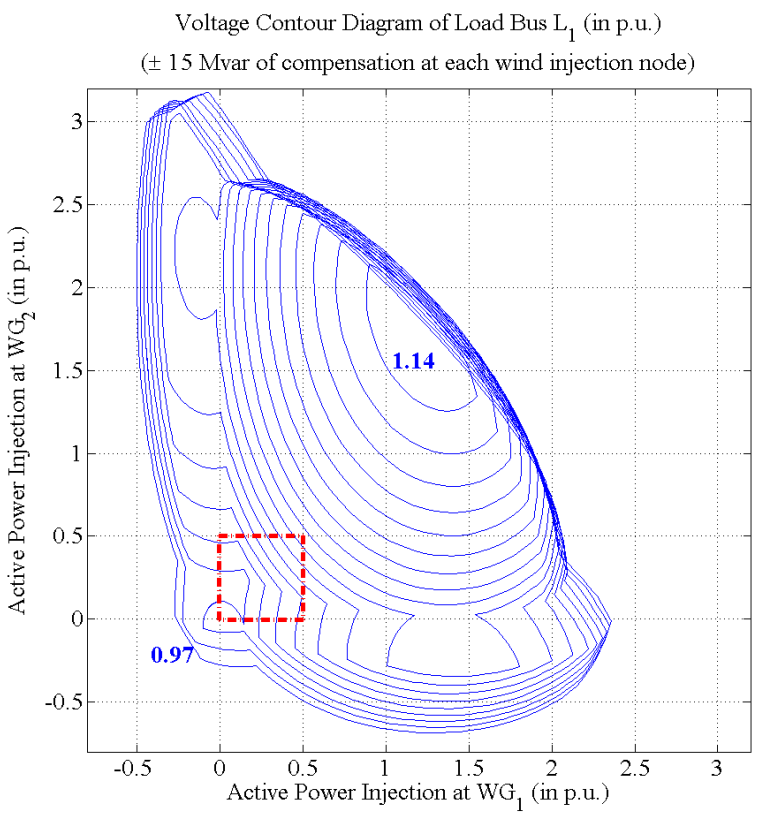

(a) Global

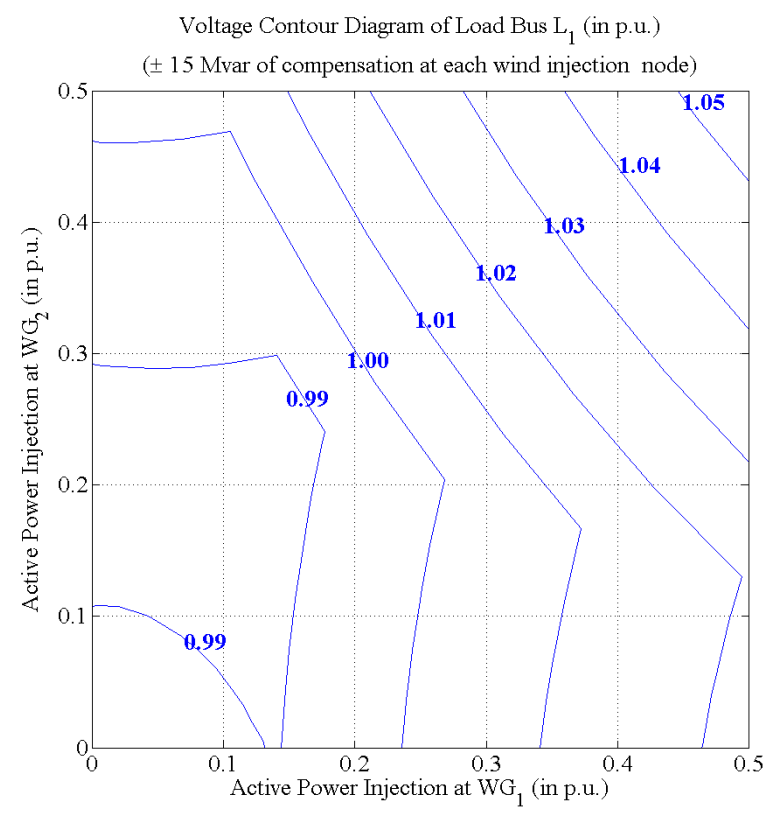

(b) Region of Interest

Fig. 3. Contour diagrams of voltage magnitude at load bus $L_{1}$ for varying active power injections at wind nodes ( \pm 15 MVAr compensation).

The simulation models tap position as a discrete variable. The power flow is executed every minute with updated load $(P, Q)$ information for all buses in the network, and with minute-by-minute wind generation data. If the voltage of the bus that is regulated by a tap-changing transformer moves outside the deadband $\left(-\frac{1}{120},+\frac{1}{120}\right)$ p.u., centered on the voltage set-point, for two consecutive power flow executions (i.e. more than one minute) then the tap is adjusted accordingly (increased or decreased by a single step) to bring the voltage magnitude within the deadband. This reflects actual tap-changing operation, where the regulated voltage is allowed to deviate from the set-point by a certain margin for a certain 
TABLE I

TAP STEP SENSITIVITIES FOR THE TRANSFORMERS HIGHLIGHTED IN FIGURE 1. NO REACTIVE COMPENSATION AT WIND INJECTION NODES.

\begin{tabular}{|c|c|c|c|c|c|c|}
\hline \multicolumn{7}{|c|}{ Tap Step Sensitivities $\left(\frac{\text { slep }}{\text { MW injection }}\right)$} \\
\hline \multirow{2}{*}{ Operating Point } & \multirow{2}{*}{ Injection Node } & \multicolumn{5}{|c|}{ Transformer } \\
\hline & & $T_{1}$ & $T_{2}$ & $T_{3}$ & $T_{4}$ & $T_{5}$ \\
\hline \multirow{2}{*}{ No Wind Output } & $W G_{1}$ & -0.01 & -0.03 & -0.07 & -0.09 & 0.00 \\
\hline & & -0.01 & -0.01 & -0.07 & -0.07 & 0.00 \\
\hline \multirow{2}{*}{$40 \%$ Wind Output } & $W G_{1}$ & 0. & -0.01 & -0.02 & -0.03 & 0.01 \\
\hline & & 0. & 0.00 & -0.02 & -0.02 & 0.02 \\
\hline \multirow{2}{*}{$80 \%$ Wind Output } & $W G_{1}$ & 0.02 & 0.00 & 0.00 & -0.01 & 0.03 \\
\hline & & 0 & 0 & 0.00 & 0 & 0.03 \\
\hline
\end{tabular}

TABLE II

TAP STEP SENSITIVITIES FOR THE TRANSFORMERS HIGHLIGHTED IN FIGURE 1. COMPENSATION OF \pm 15 MVAR AT EACH WIND INJECTION NODE.

\begin{tabular}{|c|c|c|c|c|c|c|}
\hline \multicolumn{7}{|c|}{ Tap Step Sensitivities $\left(\frac{\text { step }}{M W \text { injection }}\right)$} \\
\hline \multirow{2}{*}{ Operating Point } & \multirow{2}{*}{ Injection Node } & \multicolumn{5}{|c|}{ Transformer } \\
\hline & & $T_{1}$ & $T_{2}$ & $T_{3}$ & $T_{4}$ & $T_{5}$ \\
\hline \multirow{2}{*}{ No Wind Output } & $W G_{1}$ & 0.04 & 0.04 & 0.10 & 0.11 & 0.07 \\
\hline & $W G_{2}$ & 0.05 & 0.04 & 0.14 & 0.09 & 0.06 \\
\hline \multirow{2}{*}{$40 \%$ Wind Output } & $W G_{1}$ & 0.04 & 0.04 & 0.09 & 0.05 & 0.05 \\
\hline & & 0.08 & 0.07 & 0.20 & 0.18 & 0.14 \\
\hline \multirow{2}{*}{$80 \%$ Wind Output } & $W G_{1}$ & 0.03 & 0.01 & 0.02 & 0.03 & 0.05 \\
\hline & $W G_{2}$ & 0.03 & 0.01 & 0.02 & 0.03 & 0.06 \\
\hline
\end{tabular}

TABLE III

NUMBER OF MONTHLY TAP CHANGE OPERATIONS FOR THE TRANSFORMERS HIGHLIGHTED IN FIGURE 1.

\begin{tabular}{|c|c|c|c|c|c|}
\hline \multicolumn{5}{|c|}{ Number of Monthly Tap Changes } \\
\hline \multirow{2}{*}{ Simulation Cases } & $T_{1}$ & $T_{2}$ & $T_{3}$ & $T_{4}$ & $T_{5}$ \\
\hline No Wind & 140 & 23 & 98 & 85 & 173 \\
\hline$W G_{1}-/+15$ Mvar & 140 & 30 & 116 & 107 & 208 \\
\hline$W G_{2}^{-/+15}$ Mvar & 142 & 44 & 154 & 102 & 230 \\
\hline$W G_{1}$ and $W G_{2}$ no compensation & 142 & 28 & 134 & 113 & 176 \\
\hline$W G_{1}$ and $W G_{2}$ each -/+15 Mvar & 213 & 105 & 511 & 456 & 442 \\
\hline
\end{tabular}

period of time (typically one minute or up to several minutes depending on the setting of the transformer) before the tapchange operation is triggered. This mechanism is designed to prevent small disturbances from triggering unnecessary tapchange operations.

The cases considered in the simulation study are as follows:

1) No Wind Injection.

2) Wind Injection at $W G_{1}$ (50 MW, \pm 15 MVAr compensation).

3) Wind Injection at $W G_{2}$ (50 MW, \pm 15 MVAr compensation).

4) Wind Injection at $W G_{1}$ and $W G_{2}$ (each $50 \mathrm{MW}$, no reactive compensation).

5) Wind Injection at $W G_{1}$ and $W G_{2}$ (each $50 \mathrm{MW}, \pm 15$ MVAr compensation).

The simulations used load data for the south-east Michigan grid, and wind generation data from two wind farms in Michigan, all with one minute resolution. Table III shows the number of tap changes per month for the five cases. It can be seen that wind injection in general increases tap change operation. However this increase is significant when both wind-farms $W G_{1}$ and $W G_{2}$ have local reactive compensation (voltage regulation).

Deeper insights can be obtained by considering the trend in the number of monthly tap change operations as the reactive compensation capacity at wind injection nodes increases. This is shown in Figure 8. As the reactive compensation increases, the number of monthly tap operations initially remains rela-

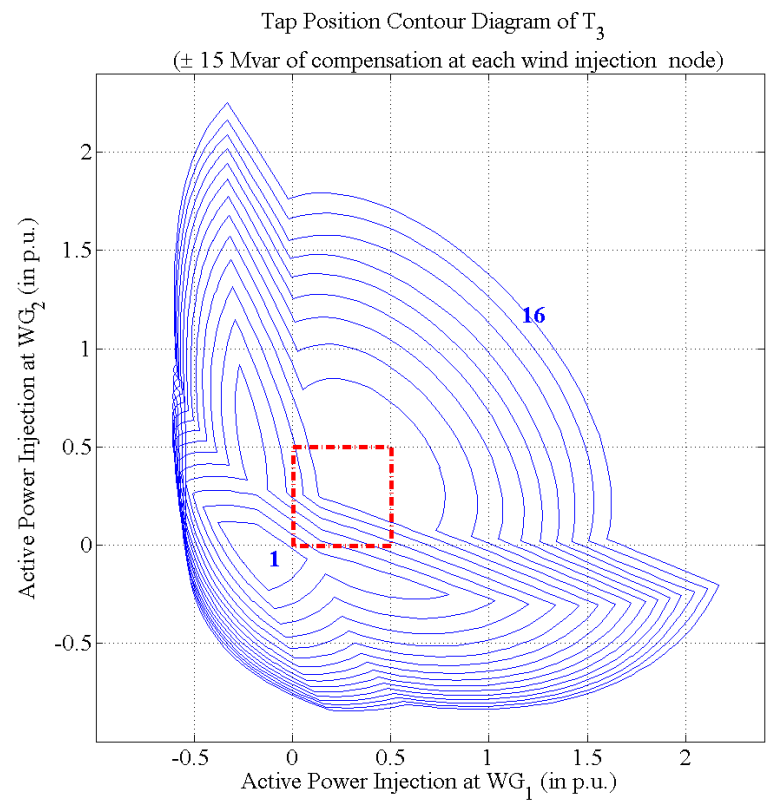

(a) Global

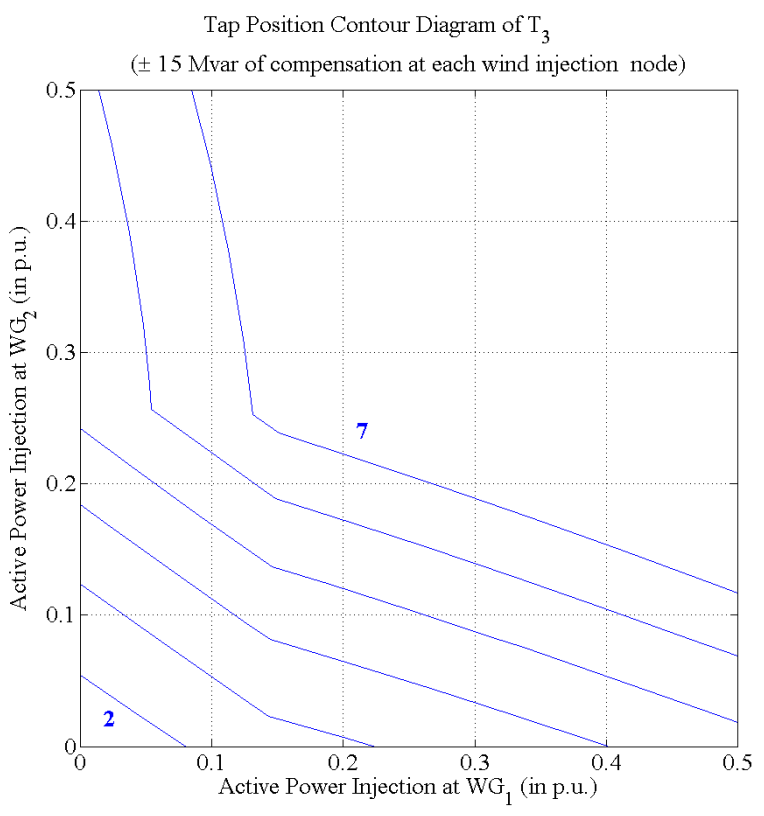

(b) Region of Interest

Fig. 4. Contour diagram of $T_{3}$ tap position for varying active power injections at wind nodes ( \pm 15 MVAr compensation).

tively constant before starting to increase significantly around \pm 10 MVAr. At that point the sub-transmission network begins to draw large amounts of reactive power from the $120 \mathrm{kV}$ transmission system. Before the voltage regulating devices reach their inductive limits (as wind injection increases), more and more reactive power is drawn from the transmission system. This increase in reactive power flow causes the voltage on the primary side of the tap-changing transformers to drop, so the transformers respond by raising taps. Figure 9 illustrates the impact of greater reactive compensation on the transmission system voltage. For higher wind power production, tighter voltage regulation at wind injection nodes and nearby load buses comes at the cost of greater voltage deviations on the 


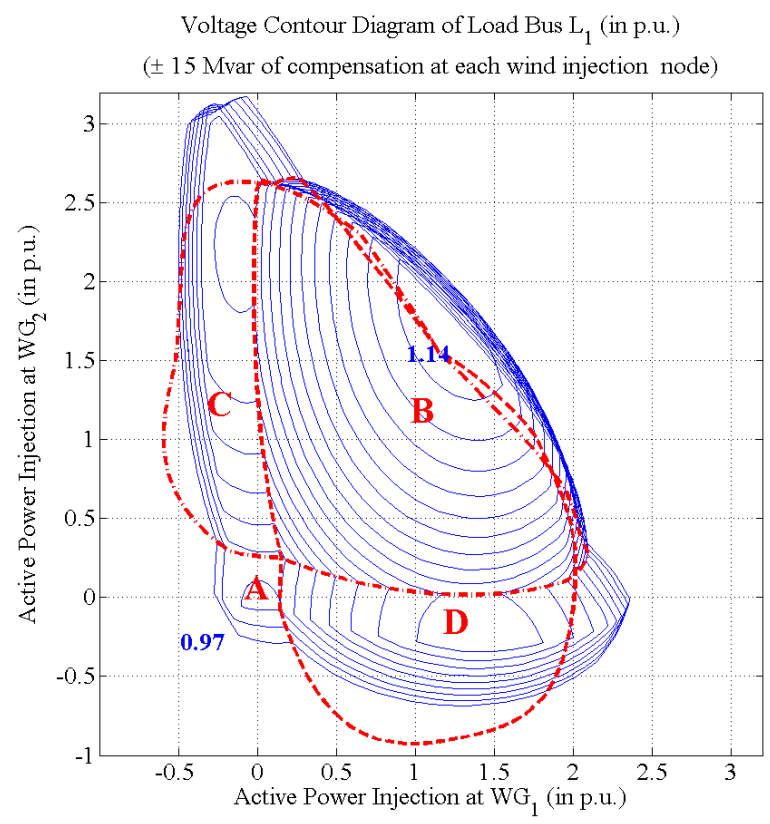

(a) Global

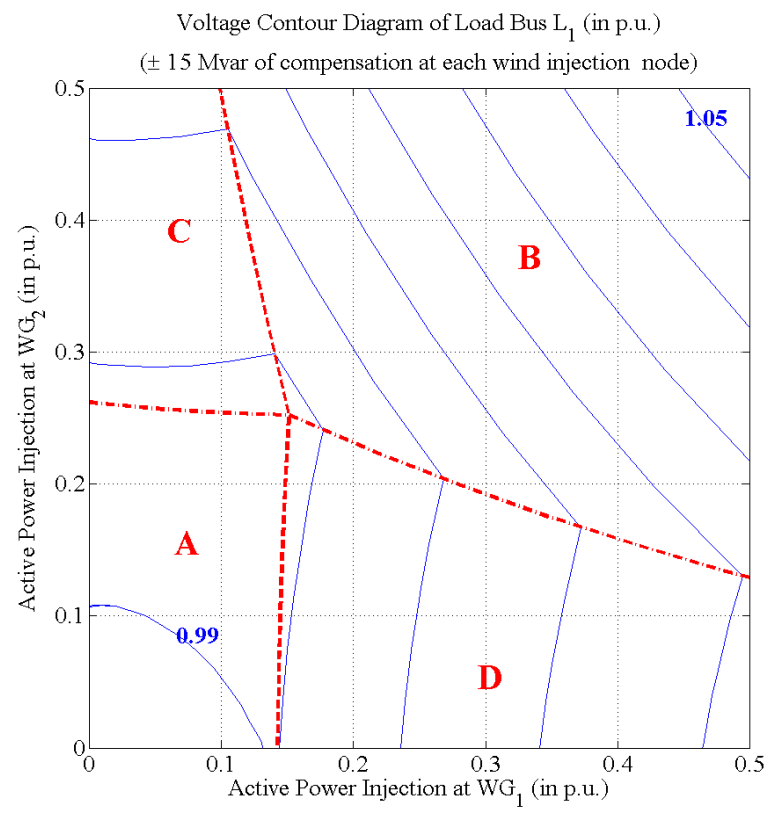

(b) Region of Interest

Fig. 5. Demarcation of the $L_{1}$ voltage magnitude contour diagram into the four regions determined by reactive compensation limits.

$120 \mathrm{kV}$ transmission system.

\section{Conclusion}

Although voltage regulation at the point of wind interconnection is indispensable, the analysis presented in the paper suggests that it has a detrimental effect on tap-changer operation for the transformers that connect the sub-transmission network $(40 \mathrm{kV})$ to the transmission system $(120 \mathrm{kV})$. Windfarm voltage regulation tends to increase the sensitivity of tap position to wind power variations, and significantly increases the number of tap change operations. Consequently, the life

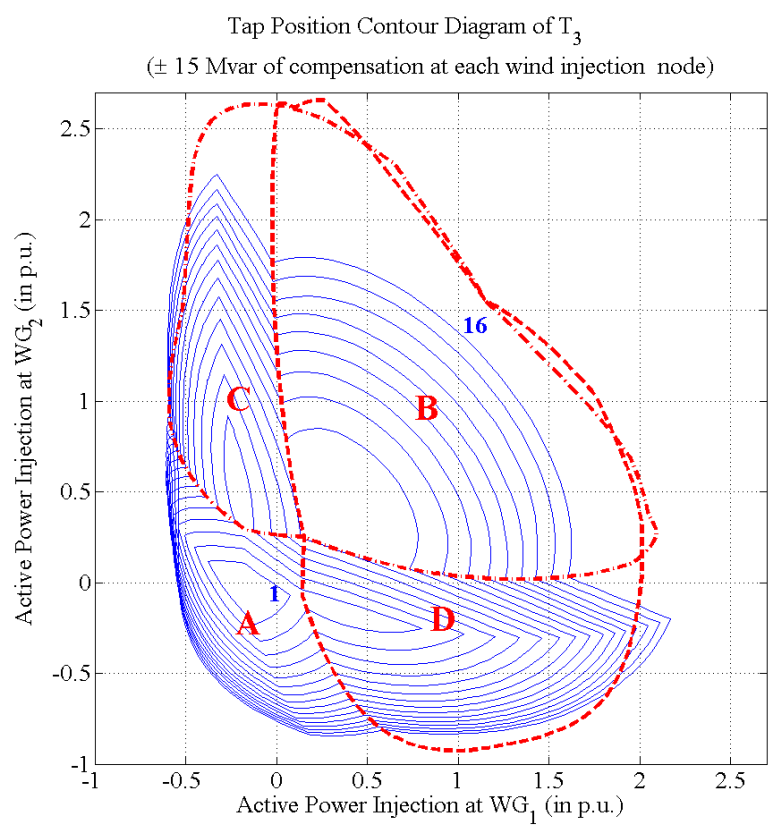

(a) Global

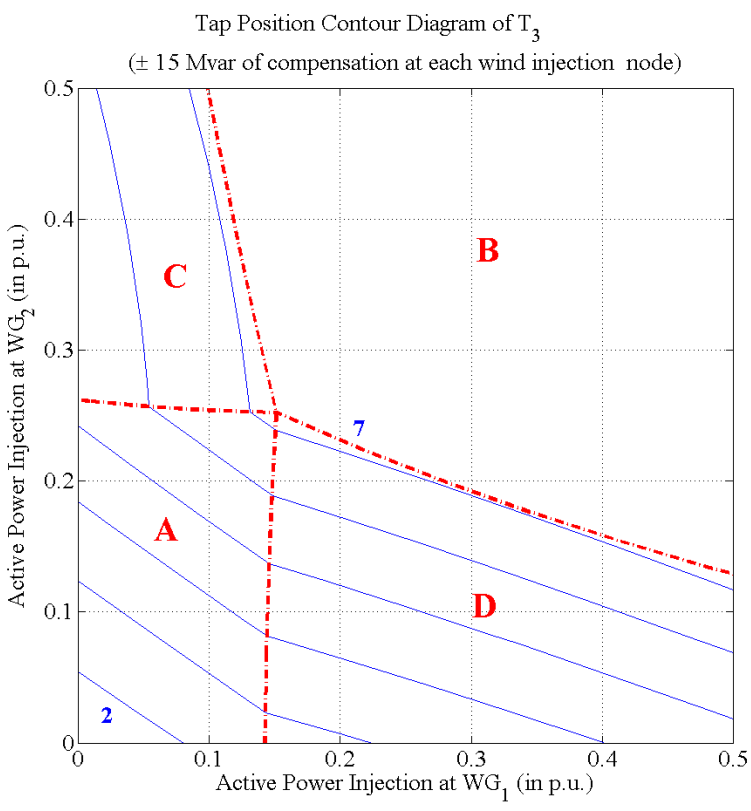

(b) Region of Interest

Fig. 6. Demarcation of the $T_{3}$ tap position contour diagram into the four regions determined by reactive compensation limits.

of these expensive assets will be decreased, hindering the development of wind power in weak grids.

The simulation results presented in the paper indicate that the tap positions of the $120 / 40 \mathrm{kV}$ transformers tend to remain relatively constant when the voltage at wind injection nodes in not regulated. On the other hand, lack of voltage regulation at wind farms leads to unacceptable voltage fluctuations within the sub-transmission network. This highlights the importance of optimizing the size of reactive compensation to balance voltage fluctuations and tap changing. 


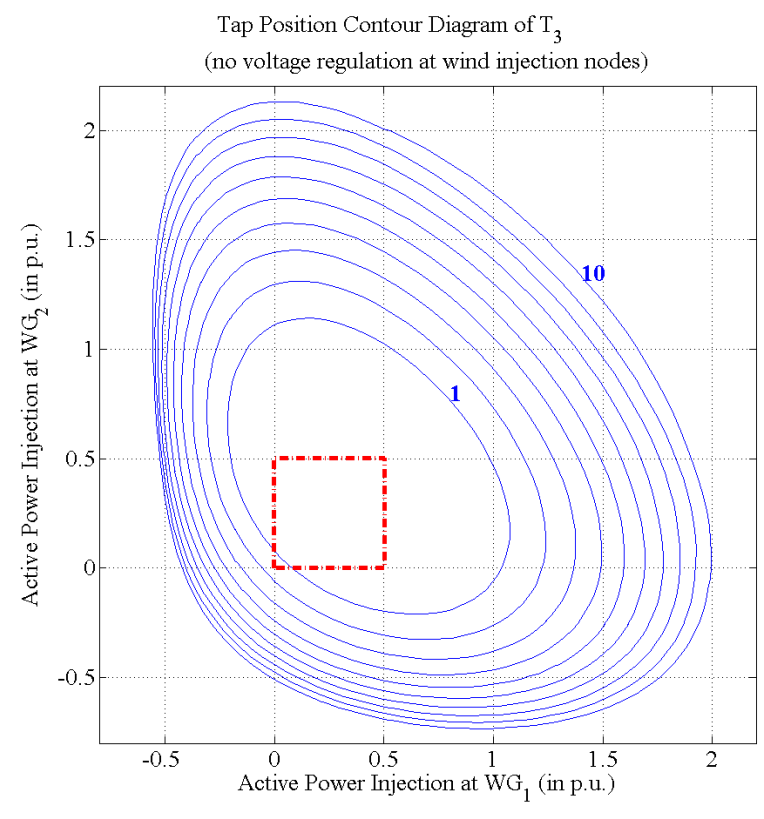

Fig. 7. Contour diagram of $T_{3}$ tap position for varying active power injections at wind nodes (no voltage regulation).

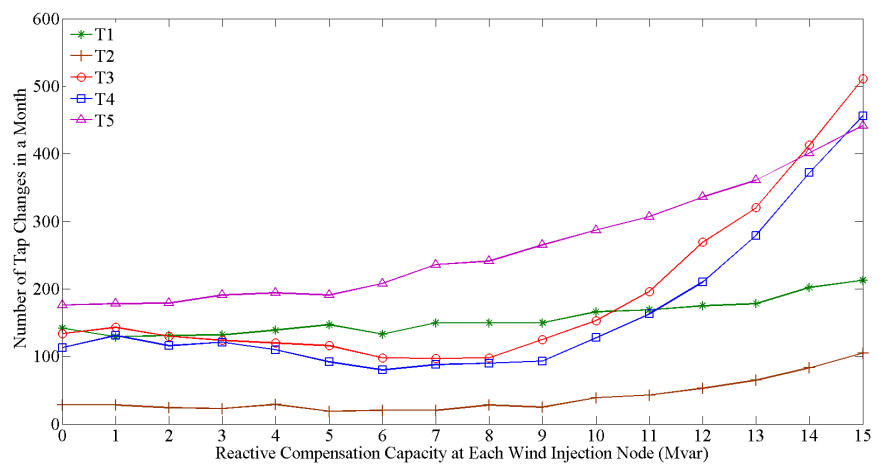

Fig. 8. Number of monthly tap-change operations for each transformer, for varying reactive compensation capacity at wind injection nodes.

\section{REFERENCES}

[1] C.L. Wadhwa, Electrical power systems, 4th Edition, New Age Publishers, 2006, pp. 727-728.

[2] P.M.S. Carvalho, P.F. Correia and L.A.F. Ferreira, "Distributed reactive power generation control for voltage rise mitigation in distribution networks", IEEE Transactions on Power Systems, vol. 23, no. 2, May 2008 , pp. 766-772.

[3] J. Peschon, D.S. Piercy, W.F. Tinney and O.J. Tveit, "Sensitivity in power systems", IEEE Transactions on Power Apparatus and Systems, vol. PAS87, no. 8, August 1968, pp. 1687-1696.

[4] M.M. Begovic and A.G. Phadke, "Control of voltage stability using sensitivity analysis", IEEE Transactions on Power Systems, vol. 7, no. 1, February 1992, pp. 114-123.

[5] N. Flatabo, O. Fosso, R. Ognedal, and T. Carlsen, "A method for calculation of margins to voltage instability applied on the Norwegian system for maintaining required security level", IEEE Transactions on Power Systems, vol. 8, no. 3, August 1993, pp. 920-928.

[6] F. Capitanescu, T. Van Cutsem, "Unified sensitivity analysis of unstable or low voltages caused by load increases or contingencies", IEEE Transactions on Power Systems, vol. 20, no. 1, February 2005, pp. 321-329.

[7] L. Soder, "Estimation of reduced electrical distribution losses depending on dispersed small scale energy production", in Proceedings 12th Power Systems Computation Conference, vol. 2, Zurich, Switzerland, 1996, pp. 1229-1234.

[8] C.B. Garcia and W.I. Zangwill, Pathways to Solutions, Fixed Points and Equilibria, Prentice Hall, Englewood Cliffs, NJ, 1981.

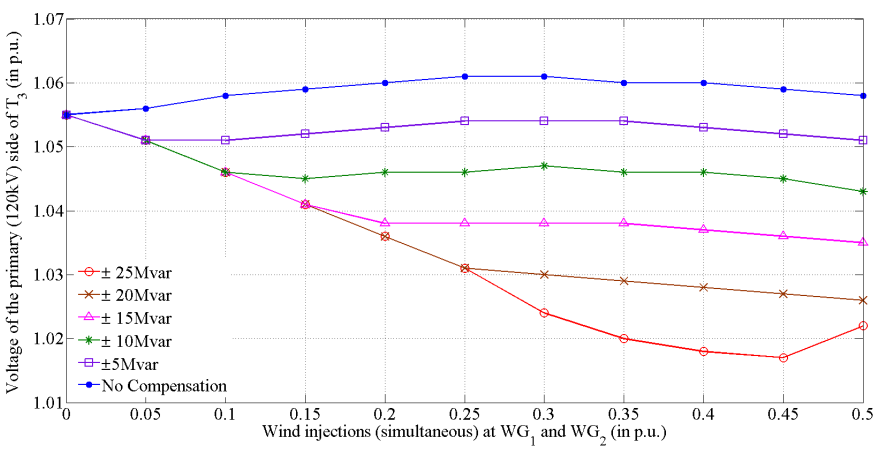

Fig. 9. Voltage drop on the primary $(120 \mathrm{kV})$ side of transformer $T_{3}$ for varying wind injection and different reactive compensation capacities.

[9] G.B. Price, "A Generalized Circle Diagram Approach for Global Analysis of the Transmission System Performance" IEEE Transaction on Power Apparatus and Systems, vol. PAS-103, no. 10, October 1984, pp. 28812890.

[10] V. Ajjarapu and C. Christy, "The continuation power flow: a tool for steady state voltage stability analysis", IEEE Transactions on Power Systems, vol. 7, no. 1, February 1992, pp. 416-423.

[11] I.A. Hiskens and R.J. Davy, "Exploring the power flow solution space boundary", IEEE Transactions on Power Systems, vol. 16, no. 3, August 2001, pp. 389-395.

[12] J.D. Glover, M.S. Sarma and T.J. Overbye, Power System Analysis and Design, 5th Edition, CL-Engineering, 2012.

[13] D.S. Bernstein, Matrix Mathematics: Theory, Facts, and Formulas, 2nd Edition, Princeton University Press, 2009.

[14] R. Seydel, Practical Bifurcation and Stability Analysis, 3rd Edition, Interdisciplinary Applied Mathematics, Vol. 5, Springer-Verlag, 1994.

[15] C.L. Masters, "Voltage rise: the big issue when connecting embedded generation to long $11 \mathrm{kV}$ overhead lines", IEE Power Engineering Journal, vol. 16, no. 1, February 2002, pp. 5-12.

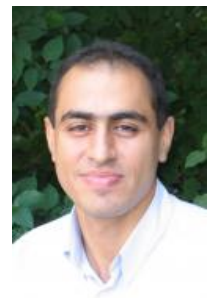

Sina Sadeghi Baghsorkhi (Student, IEEE) received the B.S. degree in electrical engineering from Sharif University of Technology, Tehran, Iran, in 2007 and the M.S. degree in natural resources and environment from the University of Michigan, Ann Arbor, MI, in 2009. Currently, he is working toward his Ph.D. degree in the Department of Electrical Engineering and Computer Science at the University of Michigan. His research interests are power system analysis, control and optimization, particularly as related to the integration of intermittent renewable generation. Since 2010 he has been involved in wind studies at the Detroit Edison Company (DTE) in Michigan. He is currently studying the impact of wind power on sub-transmission and distribution networks, and in particular is working on the applications of convex optimization methods to line congestion and voltage control/regulation issues arising from integration of large-scale wind power.

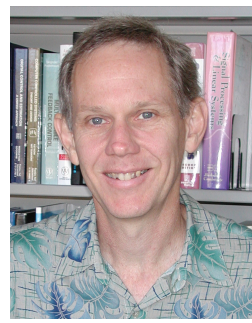

Ian A. Hiskens (Fellow, IEEE) received the B.Eng. degree in electrical engineering and the B.App.Sc. degree in mathematics from the Capricornia Institute of Advanced Education, Rockhampton, Australia, in 1980 and 1983 respectively, and the Ph.D. degree in electrical engineering from the University of Newcastle, Australia, in 1991.

$\mathrm{He}$ is the Vennema Professor of Engineering in the Department of Electrical Engineering and Computer Science, University of Michigan, Ann Arbor. He has held prior appointments in the Queensland electricity supply industry, and various universities in Australia and the United States. His major research interests lie in the area of power system analysis, in particular system dynamics and control, and security assessment. His recent activity has focused largely on integration of renewable generation and controllable load.

Dr. Hiskens is actively involved in various IEEE societies, and is VPFinance of the IEEE Systems Council. He is a Fellow of the IEEE, a Fellow of Engineers Australia and a Chartered Professional Engineer in Australia. 